\title{
Upscaling of point soil moisture measurements to field averages at the $\mathrm{OPE}^{3}$ test site
}

\author{
Gabriëlle J.M. De Lannoy ${ }^{a, *}$, Paul R. Houser ${ }^{b}$, Niko E.C. Verhoest ${ }^{a}$, \\ Valentijn R.N. Pauwels ${ }^{\text {, }}$, Timothy J. Gish ${ }^{c}$
}

a Laboratory of Hydrology and Water Management, Ghent University, Coupure links 653, B-9000 Ghent, Belgium

b George Mason University and Center for Research on Environment and Water, 4041 Powder Mill Road, Suite 302, Calverton, MD 20705-3106, United States

c Hydrology and Remote Sensing Laboratory, USDA-ARS, BARC-West Beltsville, MD 20705-2350, United States

Received 1 September 2006; received in revised form 29 May 2007; accepted 13 June 2007

\author{
KEYWORDS \\ Soil moisture; \\ Representativeness; \\ Stability; \\ Scaling; \\ Variability; \\ Observation operator
}

\begin{abstract}
Summary Some techniques are proposed to limit the representativeness error of point soil moisture observations as estimates for spatial mean soil moisture in the Optimizing Production Inputs for Economic and Environmental Enhancement $\left(\mathrm{OPE}^{3}\right)$ field site. First, representative measurement locations for the spatial mean soil moisture are sought by ranking of the sensors based on the time-mean differences between the point values and the spatial mean. Next, simple statistical methods, as well as models in both the time and frequency domain are explored to scale up point measurements to field averaged soil moisture. Upscaling by a simple linear relationship and cumulative distribution function (cdf) matching generally provide the best estimates of the temporal evolution of the spatial mean soil moisture.
\end{abstract}

(c) 2007 Elsevier B.V. All rights reserved.

\section{Introduction}

The estimation of the soil moisture mean and variation over a given spatial domain based on point measurements has received considerable attention, mainly for the interpretation of remotely sensed information and for calibration and ini-

\footnotetext{
* Corresponding author. Tel.: +3209264 61 40; fax: +3209264 6236.

E-mail address: Gabrielle.DeLannoy@UGent.be (G.J.M. De Lannoy).
}

tialization of coarse-scale land surface models over large areas. Because of the large variability of soil moisture and the disparate scales of ground samples compared to satellite footprints or coarse model grid cells, spatially representative observations require either (i) interpolation or averaging of soil moisture from several locations within a grid cell, (ii) upscaling of point measurements through some transformation or (iii) sampling locations where point measurements reflect the spatial mean behaviour.

Some well known field experiments in which intensive and distributed sampling of soil moisture was performed for the 
calibration and validation of remote sensing data, are the First International Satellite Land Surface Climatology Project (ISLSCP) Field Experiment (FIFE, Charpentier and Groffman, 1992), the Southern Great Plains Hydrology Experiments (SGP, Famiglietti et al., 1999; Bindlish and Barros, 2002) and the Soil Moisture Experiments (SMEX, Jacobs et al., 2004), amongst others. Permanent soil moisture measuring networks, such as the Oklahoma Mesonet and the Soil Climate Analysis Network (SCAN) of the Natural Resources Conservation Service (NRCS) in North America are not sufficiently dense for such calibration and validation studies. In order to obtain spatial information from distributed point measurements, these measurements are typically averaged (to remove the spatial variability) or interpolated (to study the spatial variability), e.g., by using geostatistical tools like kriging or through the application of wetness indices (Barling et al., 1994) based on terrain (and at best texture) data.

To limit the observation sampling efforts, one could simply use single point measurements and an upscaling algorithm; point measurements should be representative for the area they are assumed to cover or be transformed through some kind of observation operator (Reichle and Koster, 2004; Drusch et al., 2005) to limit the representativeness error. De Lannoy et al. (2007) studied the relative merit of variational assimilation of representative site observations versus assimilation of observations from any other site for the estimation of spatial mean soil moisture. Alternatively, if the spatial variability is to be studied, then a combination of detailed distributed modeling within a spatial area and single point measurements could be considered (i.e., through data assimilation). Crow et al. (2005) discussed how observations could be used to constrain a spatially distributed land surface model for the upscaling of point measurements.

Grayson and Western (1998) investigated the existence of certain locations in catchments that consistently show the spatial mean soil moisture behavior, irrespective of the overall wetness and the pattern of soil moisture, to determine areal estimates of soil moisture based on a limited amount of point measurements. Such locations were called Catchment Averaged Soil Moisture Monitoring (CASMM) sites and are representative 'by location'. This idea was based on the concept of time stability, introduced by Vachaud et al. (1985) and has been explored to analyze soil moisture patterns over different catchments for different purposes (Gómez-Plaza et al., 2000; Van Pelt and Wierenga, 2001; Mohanty and Skaggs, 2001; Cosh et al., 2004; Jacobs et al., 2004; Martínez-Fernández and Ceballos, 2003; Martínez-Fernández and Ceballos, 2005). Chen (2006) argued that it was more appropriate to refer to rank stability instead of time stability. Time stability would suggest a similarity in temporal changes, while the focus of rank stability is rather on temporally persistent soil moisture patterns, which are expected to be influenced by soil texture, vegetation and topography.

Data taken at a representative location could be very attractive in combination with detailed spatially distributed modeling with interaction between the grid cells: it can be expected that the assimilation of point data from a rank stable site has a wider area of influence than the assimilation of data from a site that does not show the same hydrologic behavior as the rest of the catchment. The idea of rank sta- ble sites could then be used to distinguish between more and less interesting locations for data assimilation.

The objective of this study is to estimate the field averaged soil moisture at different depths either by observations that are representative by location in the intensively instrumented agricultural field (Optimizing Production Inputs for Economic and Environmental Enhancement, $\mathrm{OPE}^{3}$ ) near Washington DC, or by upscaling point data to render them representative for field averaged soil moisture through some observation operator. Except for the studies of Martínez-Fernández and Ceballos (2003), Martínez-Fernández and Ceballos (2005) and Pachepsky et al. (2005), most studies on the identification of representative sites have focussed on top layer soil moisture, because of its direct link with remotely sensed data. In this study, however, different depths were studied, because the temporal and spatial evolution of soil moisture in the top soil layer for the $\mathrm{OPE}^{3}$ field could not be easily related to the underlying temporal pattern of soil moisture, due to complex subsurface processes (De Lannoy et al., 2006). To convert point measurements to field averaged soil moisture, some statistical methods were explored, including a time-mean bias correction, a linear transformation and cumulative density function (cdf) matching. Also models or filters identified in the frequency and time domain have been studied.

In the next section, the data are described, after which representative soil moisture sites are sought in the studied field. Then, methods are discussed to scale up the point measurements to field averaged soil moisture. Finally, the conclusions from this study are summarized.

\section{Data description}

\section{$\mathrm{OPE}^{3}$ field}

The Optimizing Production Inputs for Economic and Environmental Enhancement (OPE ${ }^{3}$, http://hydrolab.arsusda.gov/ ope3/) project is an interdisciplinary research project started in 1998 and managed by the Beltsville Agricultural Research Center (BARC) - Agricultural Research Service (ARS) of the United States Department of Agriculture (USDA). The project is conducted on a 21 ha corn field, subdivided into 4 sub-fields. The site is situated in Prince Georges County, Maryland, USA, and it is part of the Anacostia watershed. The 4 sub-watersheds are named $A, B, C$ and D from North to South.

The average height of the terrain is about $40 \mathrm{~m}$ a.s.l. and the slope is varying from $1 \%$ to $4 \%$. The top soil layer texture is sandy loam according to the USGS soil classification, with an average of $15.62 \pm 1.63 \%$ clay, $22.19 \pm 4.07 \%$ silt and $62.17 \pm 5.56 \%$ sand. A clay layer is present under the entire site, varying from $0.9 \mathrm{~m}$ to $3.5 \mathrm{~m}$ below the soil surface (Gish et al., 2002) and at some depths the soil layers contain coarse sand with abundant gravel. During summer, corn is grown on the $\mathrm{OPE}^{3}$ field, while for the studied period of May 1, 2001 through April 30, 2002, no crop was present in the winter.

\section{Soil moisture measurements}

Fig. 1 gives an overview of the field and the lay-out of the measuring sensors. In each sub-watershed, 12 capacitance 

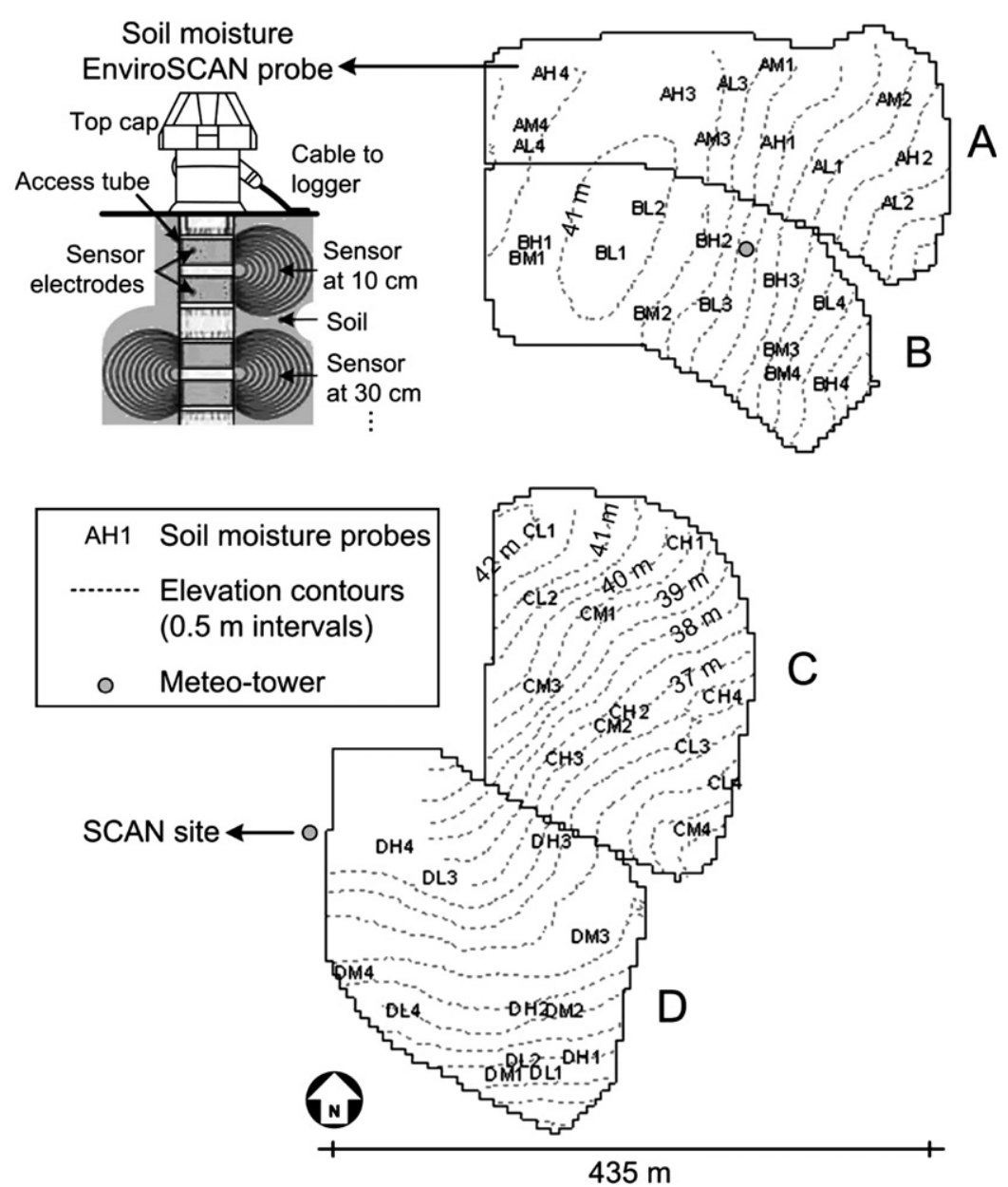

Figure 1 Digital elevation model with location of the soil moisture probes in the $\mathrm{OPE}^{3}$ field. The SCAN site is situated just upslope of field D.

probes (EnviroSCAN, SENTEK Pty Ltd., South Australia) provide soil moisture data every $10 \mathrm{~min}$. The observations were aggregated to hourly time steps. The probes were named following a three digit system. The first letter represents the name of the sub-watershed $(A, B, C, D)$, the second letter $(L, H, M)$ refers to the estimated infiltration rate at the point of installation (Low, High, Moderate clay content) and the third digit $(1,2,3,4)$ discerns between the different probes of a specific infiltration regime (Gish et al., 2002). $H$ probes have sensors at 10,30 and $80 \mathrm{~cm}$. $L$ and $M$ probes have sensors at $10,30,50,120,150$ and $180 \mathrm{~cm}$. L probes have an additional sensor at $80 \mathrm{~cm}$ depth. During the studied period, probes AL3, AL4, AM3, AM4, AH3, AH4, CL3, CL4, $\mathrm{CM} 3, \mathrm{CM} 4, \mathrm{CH} 3$ and $\mathrm{CH} 4$ were not operational because of technical defects (hit by lightning), causing that 36 out of 48 probes remained operational. A detailed analysis of the four-dimensional soil moisture data (Gish et al., 2002; De Lannoy et al., 2006) revealed a complex subsurface hydrology, mainly caused by an irregular shaped clay layer underlying the top soil layers.

Because there is a (Powder Mill) SCAN site present at the border of field $D$, these were also included in this study to make them representative for the $\mathrm{OPE}^{3}$ field soil moisture. At this site, Stevens-Vitel Hydra probes (Stevens Water
Monitoring Systems, Inc.) measure soil moisture at five different depths: 5, 10, 20, 50 and $100 \mathrm{~cm}$.

\section{Rank stable sites in the $O \mathrm{PE}^{3}$ field}

A good selection of point measurement locations can be a simple key to limit the representativeness error in point soil moisture observations, when they are needed for comparison or merging with coarser scale soil moisture estimates. Based on the idea of temporal persistence of spatial soil moisture patterns, one could seek to identify a location which is most representative for a larger area over a long time period.

\section{Method}

With $\mathrm{SM}_{j, i}$ the soil moisture for a single point or sensor $j$ at time step $i$, and $\overline{S M}_{i}$ the spatially averaged soil moisture at time step $i$, the relative difference $d_{j, i}^{r}$ of the soil moisture content for a sensor is calculated by (Vachaud et al., 1985; Grayson and Western, 1998):

$d_{j, i}^{r}=\frac{\mathrm{SM}_{j, i}-\overline{\mathrm{SM}}_{i}}{\overline{\mathrm{SM}}_{i}}$. 
The time averaged relative difference, $\left\langle d_{j}^{r}\right\rangle$, gives an indication of how much the sensor deviates from the spatial mean during a studied time period. A representative site can be identified as one for which $\left\langle d_{j}^{r}\right\rangle$ is close to 0 . A small temporal standard deviation, $\operatorname{stdv}\left(d_{j}^{r}\right)$, in the relative difference $d_{j, i}^{r}$ for a sensor $j$ implies that this sensor shows a similar temporal evolution in soil moisture as the spatial mean soil moisture. A sensor with this feature is called time or rank stable and can be used as representative for the areal soil moisture, if the offset $\left\langle d_{j}^{r}\right\rangle$ between the areal soil moisture and the soil moisture at the sensor is known. The most attractive representative site would be one for which both $\left\langle d_{j}^{r}\right\rangle$ and $\operatorname{stdv}\left(d_{j}^{r}\right)$ are close to 0 , or in other words for which the root mean square difference between the time series of point measurements and the spatial mean values is minimal.

\section{Results}

For observations at various soil depths, Fig. 2 shows the ranking of the sensors based on the one-year mean relative differences $\left\langle d_{j}^{r}\right\rangle$ between the point measurements and the entire $\mathrm{OPE}^{3}$ field soil moisture. The mean differences and their standard deviation were calculated only for those time steps for which data from a maximum of one sensor per depth in het horizontal space was missing.
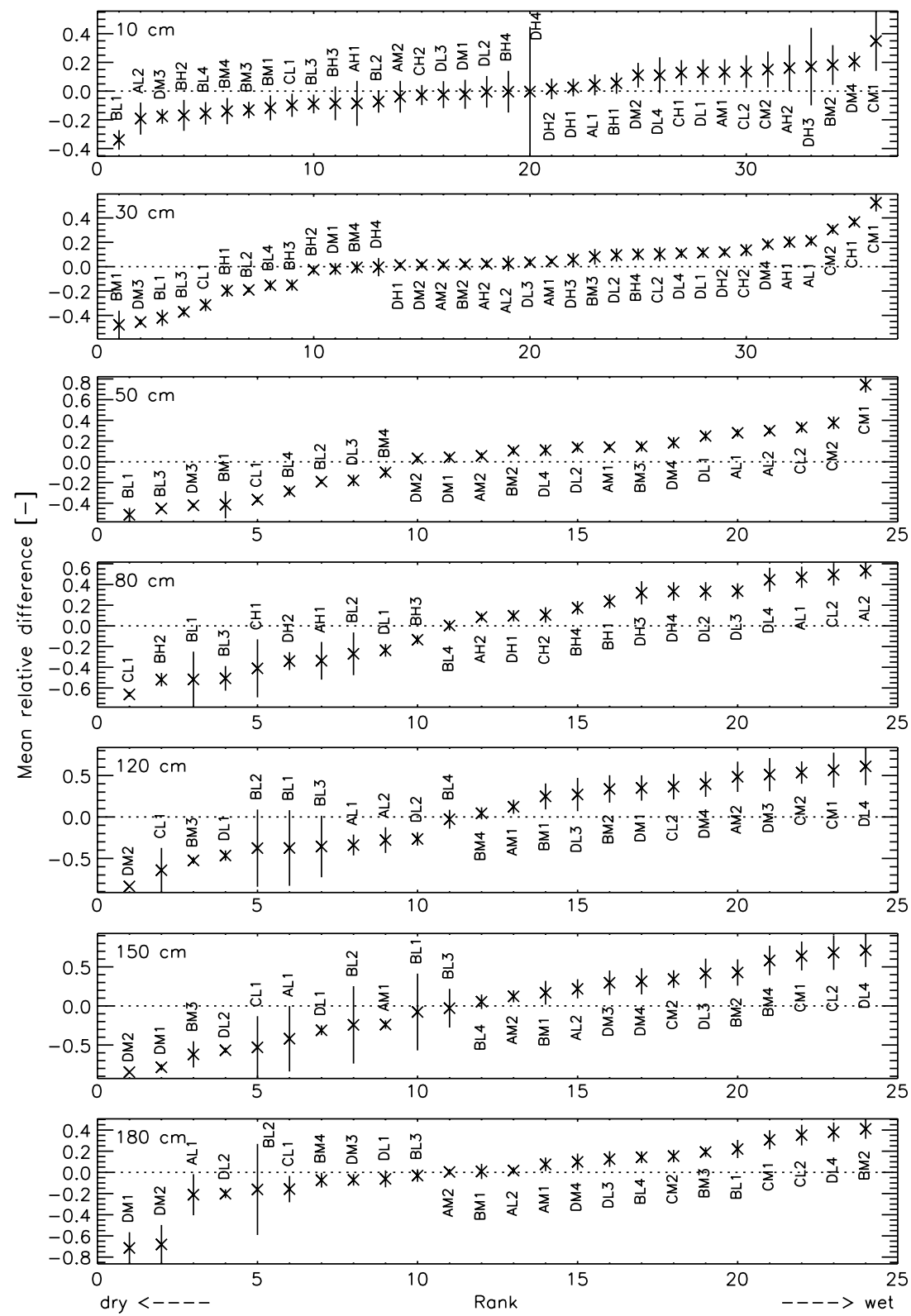

Figure 2 Average and standard deviation in relative differences between the areal wetness and point soil moisture measurements for all sensors in the entire $\mathrm{OPE}^{3}$ site. The sensors were sorted following their average deviation from the areal wetness during the one-year period from May 1, 2001 through April 30, 2002. The average deviations were calculated by almost (but not exactly) the same number of samples in time for every sensor, i.e., about 8550 . For the C-sensors there were less samples, i.e., about 7900 and for $\mathrm{AH} 2$ there were only about 5400 samples. 
For each individual observation depth in Fig. 2 one could select a sensor with a low $\left\langle d_{j}^{r}\right\rangle$ or $\operatorname{stdv}\left(d_{j}^{r}\right)$ as being representative for the field average plus or minus a constant value. For example, sensors in probes $\mathrm{DH} 1$ and $\mathrm{DH} 2$ were very close to the areal mean at $10 \mathrm{~cm}$ depth and were characterized by differences of limited variance. It was difficult to find a probe that had a small $\operatorname{stdv}\left(d_{j}^{r}\right)$ over all depths for the studied time period, but one could propose probes AM1 and BL4 for the deeper profiles and DH1 for the shallow profiles. When considering all depths, examples of probes that are not representative for the spatial mean behavior, as they had high values for $\operatorname{stdv}\left(d_{j}^{r}\right)$, are, e.g., probes DH4, BL1 and BL2. The deviant behavior of these probes could be attributed to the fact that they are situated at the highest elevations of the watershed. However, note that DH4 (near the top) had a very low $\left\langle d_{j}^{r}\right\rangle$ value in the 2 upper layers. Probes $\mathrm{BH} 1$ and $\mathrm{AH} 2$ could be proposed for shallow profiles and probes AM2 and DL3 for deep profiles as probes with overall the smallest $\left\langle d_{j}^{r}\right\rangle$ values at most depths. Soil moisture values at probe CM1 and DM2 were most different from the spatial mean soil moisture: CM1 was too wet, whereas DM2 was too dry to be representative. No significant relationship could be obtained between $\operatorname{stdv}\left(d_{j}^{r}\right)$ or $\left\langle d_{j}^{r}\right\rangle$ and the texture, mean wetness conditions, elevation or topographic index at each sensor location, probably due to the complex $\mathrm{OPE}^{3}$ hydrogeology (De Lannoy et al., 2006) at the small scale, which largely influences the soil moisture spatial structure and temporal variability. One could also argue that the structured variability in terrain features within the small field is too limited (i.e., close to random) to retrieve clear relationships with the time stability of observation locations.

Fig. 3 shows scatter plots which compare $\left\langle d_{j}^{r}\right\rangle$ and $\operatorname{stdv}\left(d_{j}^{r}\right)$ in the first ('summer': May 1, 2001 through October 31, 2001) and second ('winter': November 1, 2001 through April 30, 2002) half year. The mean relative difference $\left\langle d_{j}^{r}\right\rangle$ and hence the ranking of the sensors per depth was well preserved through time. The value of $\operatorname{stdv}\left(d_{j}^{r}\right)$ was clearly larger in the summer than in the winter period, which corresponds to the observation that the spatial variability reached maximal values in the spring-summer of 2001 (De
Lannoy et al., 2006). Consequently, when a sensor with a low value of $\left\langle d_{j}^{r}\right\rangle$ is found for one period, then one could be reasonably confident that the estimation of the spatial mean in another period would not be significantly biased. On the other hand, if one selects a sensor with a low $\operatorname{stdv}\left(d_{j}^{r}\right)$ value for one period to assess the spatial mean, then there is no guarantee that the temporal variability of the spatial mean in another period would be accurately estimated. It should be remarked that mainly for the upper layers in periods with a high frequency of precipitation events and in summer time, some individual sensors were switching ranks in time (not shown), indicating that during these periods the spatial soil moisture pattern may not be exactly persistent.

The ranking of the mean temporal cross-correlation of a sensor with all other sensors at the same depth revealed that for larger values of $\operatorname{stdv}\left(d_{j}^{r}\right)$, a lower mean temporal cross-correlation with all other sensors can be expected in the upper layers, as illustrated in Fig. 4. Since in the OPE ${ }^{3}$ field the spatial correlation length was found to be longer for higher mean cross-correlation (De Lannoy et al., 2006), this also indicates that a smaller $\operatorname{stdv}\left(d_{j}^{r}\right)$ corresponds to a larger correlation length. If a land surface model correctly simulates the spatial structure through its dynamics, then state updating through assimilation of observations at points with a large soil moisture correlation length (which might occur where $\operatorname{stdv}\left(d_{j}^{r}\right)$ is low) is expected to have more impact than soil moisture assimilation at a point where the soil moisture is only marginally correlated with the surrounding points. However, to be truly useful for assimilation, the representative points should also have a low $\left\langle d_{j}^{r}\right\rangle$, i.e., a limited bias. This can be achieved after a proper transformation (see below).

\section{Observation upscaling operators}

To estimate spatially averaged soil moisture from point data, e.g., for direct validation of remote sensing products or assimilation of the point data into a coarse grid assimilation system, it is beneficial to scale up the point data by an existing relationship. Even if these data were collected at a
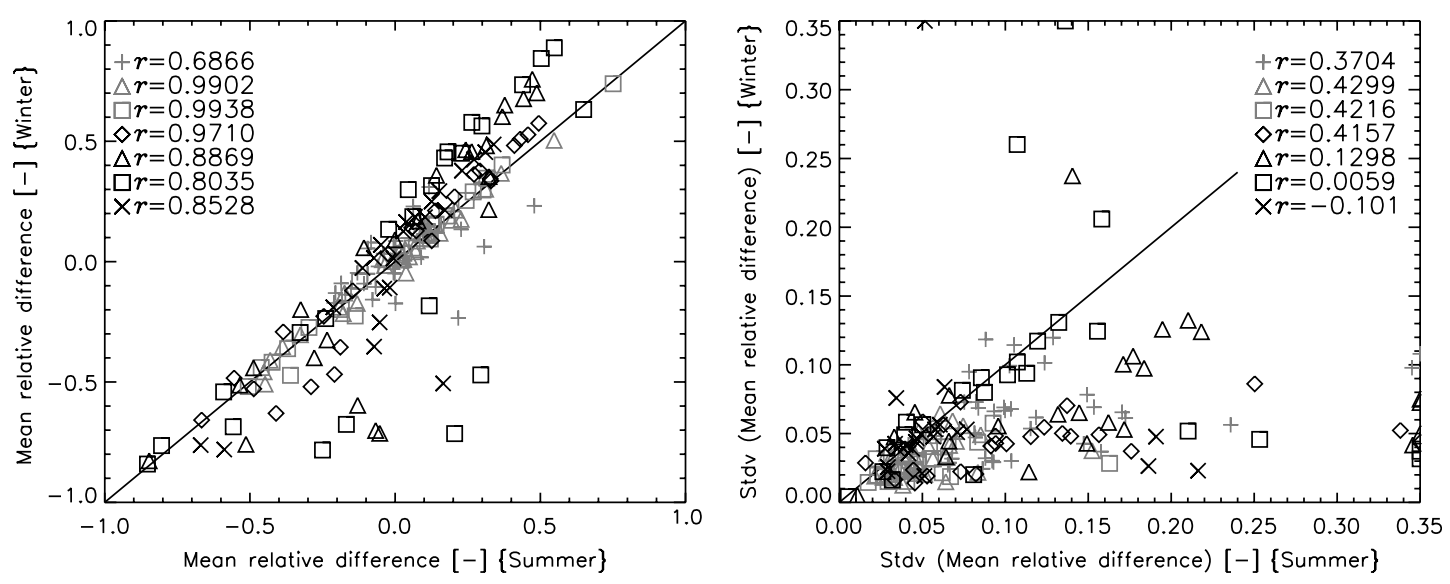

Figure 3 Relation between the ranking of the relative $\left\langle d_{j}\right\rangle$ and $\operatorname{stdv}\left(d_{j}\right)$ in the first and second half year of observations. Gray $(+)$, $(\triangle)$ and $(\square)$ are for 10,30 and $50 \mathrm{~cm}$ depth. Black $(\diamond),(\triangle),(\square)$ and $(\times)$ are for $80,120,150$ and $180 \mathrm{~cm}$ depth. $r$ is the correlation coefficient. 


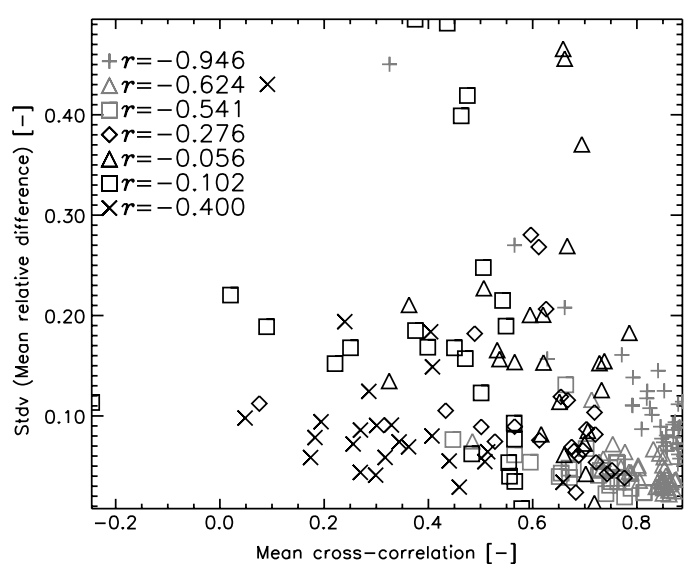

Figure 4 Relation between the $\operatorname{stdv}\left(d_{j}\right)$ and the mean correlation with all surrounding sensors in a same soil layer for the 1 year of observations. Gray $(+),(\triangle)$ and $(\square)$ are for 10 , 30 and $50 \mathrm{~cm}$ depth. Black $(\diamond),(\triangle),(\square)$ and $(\times)$ are for 80, 120, 150 and $180 \mathrm{~cm}$ depth. $r$ is the correlation coefficient of the possible relationships.

representative site showing the same behavior as the field average, there might be for example a constant offset between those data and the field average at some soil depths. For the purpose of this upscaling, time-invariant relationships were studied. Single-input-single-output, constant parameter models or observation operators were identified to convert the one-year point data time series for the individual sensors (input) to the field averaged time series of soil moisture (output). For assimilation purposes, it is important to realize that the use of point observations for spatial mean purposes increases the uncertainty of the observational information and may even introduce bias. An upscaling operation will improve the overall precision of the observations, but at the expense of an increased uncertainty (the upscaling will never be perfect).

\section{Instantaneous statistical corrections in the time domain}

The first class of methods provides instantaneous relationships to scale up point measurements as they become available. A conversion of the point data $u_{j, i}=S M_{j, i}$ to the estimated field averaged soil moisture $\hat{y}_{i}=\widehat{S M}_{i}$ can be achieved through the use of the time mean relative difference, with $d_{j, i}^{r}$ (Eq. 1) replaced by its time average, $\left\langle d_{j}^{r}\right\rangle$ :

$\hat{y}_{i}=\frac{u_{j, i}}{\left\langle d_{j}^{r}\right\rangle+1}$

However, $\left\langle d_{j}^{r}\right\rangle$ does not provide an unbiased estimator in case of noise on the $\overline{\mathrm{SM}}_{i}$ signal and differences from the spatially averaged soil moisture have a lower weight in $\left\langle d_{j}^{r}\right\rangle$, in case the spatial averaged soil moisture is high (Eq. 1). Furthermore, through the multiplicative relationship the amplitude of $\widehat{S M}_{i}$ will be smaller than that of $\mathrm{SM}_{j, i}$, if $\left\langle d_{j}^{r}\right\rangle$ is positive. The opposite is true for negative $\left\langle d_{j}^{r}\right\rangle$. However, no such problems are encountered by studying the absolute difference $d_{j, i}^{a}=\mathrm{SM}_{j, i}-\overline{\mathrm{SM}}_{i}$, which results in the additive relationship:

$\hat{y}_{i}=u_{j, i}-\left\langle d_{j}^{a}\right\rangle$.
The ranking of the $\mathrm{OPE}^{3}$ sensors as discussed above was very similar for relative and absolute differences (not shown). The absolute difference simply corrects the first moment of the time series probability density function (pdf).

Use of a linear relationship $\hat{y}_{i}=a+b u_{j, i}$, with $a$ and $b$ fitted constant parameters, extends this approach with one degree of freedom and is therefore expected to yield a further improved estimated field average. Because of the linear operation of these three approaches, the obtained upscaled (estimated field averaged soil moisture) time series will be equally well correlated to the observed field averaged time series.

In order to match the higher order moments of both the point and spatially averaged soil moisture data series, cumulative density function (cdf) matching (Atlas et al., 1990; Reichle and Koster, 2004; Drusch et al., 2005) was explored. As in Drusch et al. (2005), it was found that third order polynomials best fitted to the difference between spatial mean and point observations $\left(\overline{S M}_{i}-S_{j, i}\right)$ as function of the observed point data for soil layers within the rooting depth. A least-square optimization of the polynomial coefficients was performed to find the best fit. At deeper layers it was impossible to find an accurate observation operator, due to a very limited temporal variability in most periods, together with some sudden dry-out events in deeper layers at some points.

\section{Time series conversion in the frequency domain}

If no instantaneous transformation is required, then the time series of point data (input) can be passed through a filter to obtain the spatially averaged (output) time series, or alternatively the spectrum of the point data (input) can be filtered to obtain the field averaged data (output) in the frequency domain.

The spectral description of the upscaling process is given by the filter or frequency response $H(k)$, which can be found through:

$S_{u y}(k)=H(k) S_{u u}(k)$,

with $k$ a discrete frequency and $S_{u y}(k)$ and $S_{u u}(k)$ the crossand auto-spectral density functions. These functions are the Fourier transforms of the cross- and autocorrelation functions, with $u_{j, i}=\mathrm{SM}_{j, i}$ the point data and $\mathrm{y}_{i}=\overline{\mathrm{SM}}_{i}$ the observed field averaged data.

$S_{u y}$ and $S_{u u}$ were estimated by periodograms, i.e., the square of the magnitude of the Fourier transform of the time series. If the complete time series is used, then it is possible to find a filter that perfectly generates the output for the given input time series, however at the expense of a lack in generality: this specific filter would be applicable to different time periods only with a limited reliability. To increase this reliability and to develop a filter which is more generally applicable, the method of Welch (1967) with ensemble averaging was applied to obtain limited variance periodograms. The hourly one-year time series were divided into smaller records of $L=512$ hourly elements (a Hamming window was imposed to limit spectral leakage) and an ensemble average of the periodograms for these sections was calculated (Oppenheim and Schafer, 1975). For the 
close to zero frequencies (static assumption, theoretically corresponding to an infinite time scale), the retrieved upscaling functions give an indication of how much the overall input time series should be shifted to get a good fit with the observed output, i.e., to overcome the time mean discrepancy between the two time series. The amplitude ratio at frequencies close to zero approached 1. For most sensors within the rooting depth, the amplitude ratio decreased with increasing frequencies and was below 1 for high frequencies, which indicates a smaller temporal variability in the space averaged time series compared to the point data, which conforms the expectations. An example of the transfer function to convert the point data from sensor BM3 to the field average at $10 \mathrm{~cm}$ depth is shown in Fig. 5 . For this and many other sensors, it can be concluded that the spatial soil moisture can be expected to be smoother than the point data for variability at the time scale of a day and less, while the variability at larger time scales is similar for point and space averaged data.

The Fourier transform of the estimated field average soil moisture $\hat{y}_{i}=\widehat{\widehat{S M}}_{i}$ over each time interval of $L$ elements is found as:

$\hat{Y}(k)=H(k) U_{j}(k)$,

where $U_{j}(k)$ is the Fourier transformed input $u_{j, i}=\mathrm{SM}_{j, i}$ over the same time interval. Since $H(k)$ was determined for $L \ll N$, with $N$ the total length of the time series, the complete time series of $\hat{y}_{i}$ was a concatenation of small time series obtained after inverse Fourier transformation of $\widehat{Y}(k)$ resulting from small consecutive input records of length $L$. Some overlapping was included to reduce edge effects.

It should be recognized that unlike the complete pdfs, the correlation functions or spectral characteristics do not completely describe the time series. Further, the treatment in the frequency domain is only useful for the upper soil layers where periodic variations such as diurnal soil moisture variability and dynamics due to precipitation are well observed, while for the deeper soil layers there are little or no periodic components observed.

\section{Time series conversion in the time domain}

In the discrete time domain, the relation between input $\left(u_{j, i}=\mathrm{SM}_{j, i}\right)$ and output $\left(\mathrm{y}_{i}=\overline{\mathrm{SM}}_{i}\right)$ can be given by a difference equation:

$$
\begin{aligned}
& y_{i}+a_{1} y_{i-1}+\cdots+a_{n_{a}} y_{i-n_{a}} \\
& \quad=b_{0} u_{j, i}+b_{1} u_{j, i-1}+\cdots+b_{n_{b}} u_{j, i-n_{b}} .
\end{aligned}
$$

This is a general expression for a autoregressive moving average (ARMA) model, with orders $n_{a}$ and $n_{b}$ and without inclusion of a time delay. This model can be written as:

$\mathbf{y}_{i}=\mathbf{G}_{i} \hat{\boldsymbol{\theta}}+\mathbf{e}_{i}$,

with the observed output $\mathbf{y}_{i}=\left[y_{i} \cdots y_{i+N-1}\right]^{\top}$, the parameter vector to be estimated $\hat{\boldsymbol{\theta}}=\left[\begin{array}{ll}\hat{a}_{1}, \ldots, \hat{a}_{n_{a}} & \hat{b}_{0}, \ldots, \hat{b}_{n_{b}}\end{array}\right]^{\top}$, the error to minimize $\boldsymbol{e}_{i}$ and

$$
\mathbf{G}_{i}=\left[\begin{array}{cccccc}
-y_{i-1} & \cdots & -y_{i-n_{a}} & u_{j, i} & \cdots & u_{j, i-n_{b}} \\
\vdots & \vdots & \vdots & \vdots & \vdots & \vdots \\
-y_{i+N-2} & \cdots & -y_{i+N-1-n_{a}} & u_{j, i+N-1} & \cdots & u_{j, i+N-1-n_{b}}
\end{array}\right] .
$$

$N$ is the number of elements in the time series. The least square parameter estimator is found by $\hat{\boldsymbol{\theta}}=\left[\mathbf{G}_{i}^{\top} \mathbf{G}_{i}\right]^{-1} \mathbf{G}_{i}^{\top} \mathbf{y}_{i}$. By iterative searching, the best model structure (i.e., the orders $n_{a}$ and $n_{b}$ ) was sought. Because of the iteration in the identification of the best ARMA upscaling model and the recursive operation to estimate $\hat{y}_{i}$, this method was the most computational expensive of the different proposed methods. The estimated output time series $\hat{y}_{i}$ resulted from feeding $u_{j, i}$ through the estimated model, after a spinup of a few years, generated by a repetition of identical input time series $u_{j, i}$.

\section{Results}

Fig. 6 shows how point data from sensor BM1 are converted to field averaged data by different proposed methods. The Root Mean Square Error (RMSE), Nash-Suttcliffe criterium (NS), correlation $(R)$, and absolute mean difference (BIAS) were calculated as measures of goodness-of-fit between the observed $\left(y_{i}\right)$ and estimated $\left(\hat{y}_{i}\right)$ field average soil moisture:

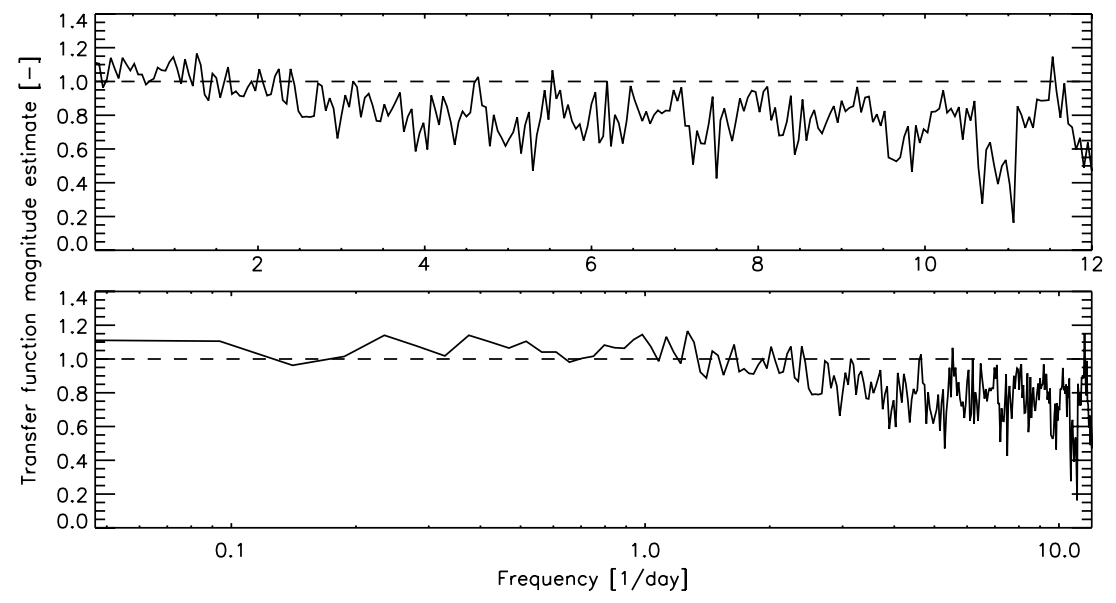

Figure 5 Estimate of the transfer function magnitude to scale up point data from sensor BM3 to the OPE ${ }^{3}$ field average soil moisture at $10 \mathrm{~cm}$ depth. The lower plot shows the same information as the upper plot, but at a logarithmic frequency scale to zoom into the low frequencies. The Nyquist frequency (the maximum plotted frequency) is half of the sampling frequency, i.e., 1 per $2 \mathrm{~h}$. 


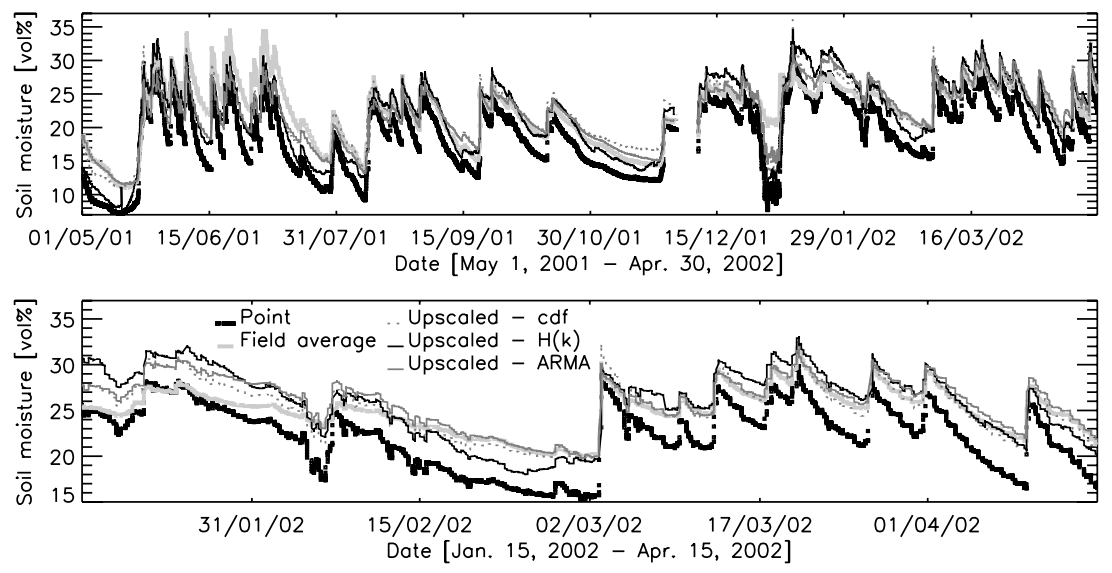

Figure 6 Estimated $\mathrm{OPE}^{3}$ field averaged soil moisture at $10 \mathrm{~cm}$ based on point data (black squares) from sensor BM1 after upscaling through cdf-matching (dashed black line), filtering in the frequency domain (full black line) and filtering with an ARMA model (full gray line). The gray squares are observed field averages. The lower plot shows the detail in a selected time interval.

$$
\begin{aligned}
& \text { RMSE }=\sqrt{\frac{1}{N} \sum_{i=1}^{N}\left(y_{i}-\hat{y}_{i}\right)^{2},} \\
& N S=1-\frac{\sum_{i=1}^{N}\left(y_{i}-\hat{y}_{i}\right)^{2}}{\sum_{i=1}^{N}\left(y_{i}-\langle y\rangle\right)^{2}}, \\
& R=\frac{\sum_{i=1}^{N}\left(y_{i}-\langle y\rangle\right)\left(\hat{y}_{i}-\langle\hat{y}\rangle\right)}{\sqrt{\sum_{i=1}^{N}\left(y_{i}-\langle y\rangle\right)^{2} \sum_{i=1}^{N}\left(\hat{y}_{i}-\langle\hat{y}\rangle\right)^{2}}} \\
& \text { BIAS }=\left|\frac{1}{N} \sum_{i=1}^{N} y_{i}-\frac{1}{N} \sum_{i=1}^{N} \hat{y}_{i}\right|=|\langle y\rangle-\langle\hat{y}\rangle|,
\end{aligned}
$$

where $N$ is the number of available data points in 1 year and notation \langle\rangle refers to temporally averaged variables. Table 1 summarizes how well the six different methods were able to reproduce the field mean $\left(\hat{y}_{i}\right)$ for some sensors at $10 \mathrm{~cm}$ depth in field $B$, given a one-year time series of hourly point observations.

For each goodness-of-fit measure, the transformation of all six methods that performed best per given point input time series was identified. Fig. 7 shows the frequency of best performance of each method for the Nash-Suttcliffe (similar for RMSE) and correlation criterium over all sensors per layer (36 or 24 active sensors depending on the depth). No information is given for soil moisture at $180 \mathrm{~cm}$ depth, because the Fourier transformation could not be performed for this almost time-invariable deep soil moisture. Fig. 7

\begin{tabular}{|c|c|c|c|c|c|c|c|c|c|c|c|c|}
\hline \multirow[b]{2}{*}{$10 \mathrm{~cm}$} & \multicolumn{6}{|c|}{ RMSE } & \multicolumn{6}{|l|}{ BIAS } \\
\hline & M1 & $M 2$ & M3 & M4 & M5 & M6 & M1 & $M 2$ & $M 3$ & M4 & M5 & M6 \\
\hline $\mathrm{BH} 1$ & 1.61 & 1.51 & 1.45 & 1.35 & 1.54 & 1.55 & 0.00 & $3.34 \mathrm{E}-02$ & $4.20 \mathrm{E}-05$ & $1.63 \mathrm{E}-04$ & $3.82 \mathrm{E}-03$ & $8.25 \mathrm{E}-02$ \\
\hline $\mathrm{BH} 2$ & 2.19 & 2.86 & 1.94 & 1.98 & 2.58 & 1.92 & 0.00 & $2.68 \mathrm{E}-01$ & $6.60 \mathrm{E}-05$ & $4.29 \mathrm{E}-03$ & $5.16 \mathrm{E}-01$ & $2.56 \mathrm{E}-01$ \\
\hline BL1 & 1.36 & 1.73 & 1.16 & 1.02 & 1.75 & 1.42 & 0.00 & $2.57 \mathrm{E}-01$ & $4.50 \mathrm{E}-05$ & $5.92 \mathrm{E}-03$ & $1.14 \mathrm{E}+00$ & $1.40 \mathrm{E}-01$ \\
\hline BL2 & 1.71 & 1.65 & 1.63 & 1.63 & 1.62 & 1.68 & 0.00 & $2.01 \mathrm{E}-01$ & $5.60 \mathrm{E}-05$ & $7.75 \mathrm{E}-02$ & $2.01 \mathrm{E}-01$ & $2.27 \mathrm{E}-01$ \\
\hline BM1 & 1.99 & 1.99 & 1.97 & 1.59 & 1.97 & 2.14 & 0.00 & $1.98 \mathrm{E}-01$ & $1.80 \mathrm{E}-05$ & $1.29 \mathrm{E}-02$ & $4.54 \mathrm{E}-02$ & $5.12 \mathrm{E}-01$ \\
\hline \multirow[t]{3}{*}{ BM2 } & 2.45 & 2.19 & 2.15 & 2.00 & 2.20 & 2.33 & 0.00 & $2.18 \mathrm{E}-01$ & $2.10 \mathrm{E}-05$ & $9.62 \mathrm{E}-03$ & $8.96 \mathrm{E}-02$ & $4.41 \mathrm{E}-01$ \\
\hline & \multicolumn{6}{|l|}{ NS } & \multicolumn{6}{|l|}{$R$} \\
\hline & M1 & $M 2$ & M3 & M4 & M5 & M6 & M1 & $M 2$ & M3 & M4 & M5 & M6 \\
\hline $\mathrm{BH} 1$ & 0.88 & 0.89 & 0.90 & 0.92 & 0.89 & 0.89 & 0.95 & 0.95 & 0.95 & 0.96 & 0.95 & 0.94 \\
\hline $\mathrm{BH} 2$ & 0.78 & 0.62 & 0.83 & 0.82 & 0.69 & 0.83 & 0.91 & 0.91 & 0.91 & 0.91 & 0.91 & 0.91 \\
\hline BL1 & 0.92 & 0.86 & 0.94 & 0.95 & 0.86 & 0.91 & 0.97 & 0.97 & 0.97 & 0.98 & 0.97 & 0.95 \\
\hline BL2 & 0.87 & 0.87 & 0.88 & 0.88 & 0.88 & 0.87 & 0.94 & 0.94 & 0.94 & 0.94 & 0.94 & 0.94 \\
\hline BM1 & 0.82 & 0.82 & 0.82 & 0.88 & 0.82 & 0.79 & 0.91 & 0.91 & 0.91 & 0.94 & 0.91 & 0.90 \\
\hline BM2 & 0.72 & 0.78 & 0.79 & 0.82 & 0.78 & 0.75 & 0.89 & 0.89 & 0.89 & 0.91 & 0.89 & 0.87 \\
\hline
\end{tabular}

Table 1 Root mean square error (RMSE, [vol.\%]), absolute mean difference (BIAS, [vol.\%]), Nash-Suttcliffe criterium (NS, [-]) and correlation $(R,[-])$ between the observed $\mathrm{OPE}^{3}$ field averaged soil moisture and upscaled point measurements from some sensors in the $B$ field at $10 \mathrm{~cm}$ over 1 year, using different methods $(M)$

$M 1$ = absolute mean difference, $M 2=$ relative mean difference, $M 3=$ linear relationship, $M 4=$ cdf-matching, $M 5=$ transfer function in frequency domain and M6 = ARMA model. 

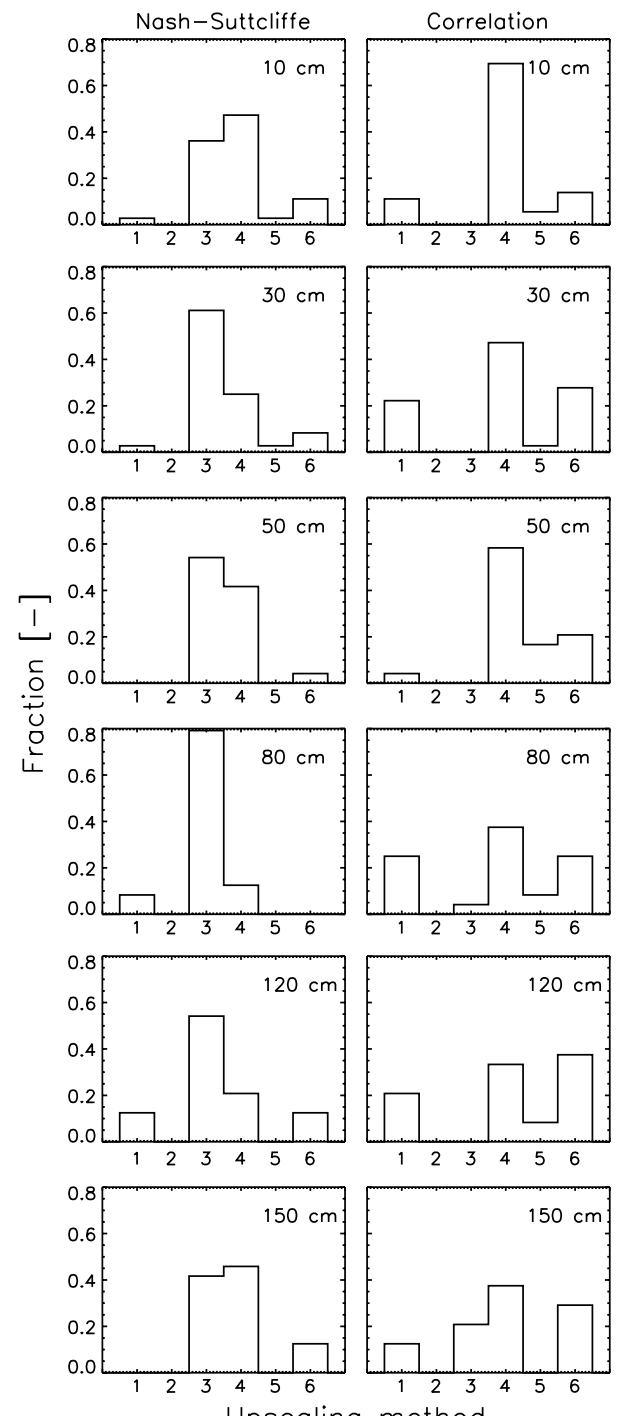

Upscaling method

Figure 7 Fraction of the methods $(M)$ resulting in a best score for the Nash-Suttcliffe and correlation measure between spatial average and upscaled point soil moisture over a 1-year period at different soil depths. $M 1$ = absolute mean difference, $M 2$ = relative mean difference, $M 3=$ linear relationship, M4 = cdf-matching, $M 5$ = transfer function in frequency domain and $M 6=$ ARMA model.

and Table 1 show that, independent of the depth, the lowest values for the RMSE and the highest values for NS were found for the linear transformation $\hat{y}_{i}=a+b u_{j, i}$ (M3) and the cdf-matching (M4). To obtain the best correlation $R$ between the observed and estimated spatial mean soil moisture, cdf-matching was clearly the best solution for the upper layers and for deeper layers also an ARMA model resulted in a high correlation measure. Obviously, the smallest (i.e., zero) BIAS between the estimated and observed spatial time series was achieved by simply adding the absolute mean $\left\langle d_{j}^{a}\right\rangle$ (M1, Table 1). However, this may not be the case when $\left\langle d_{j}^{a}\right\rangle$ would be used to upscale data which were not used in the determination of $\left\langle d_{j}^{a}\right\rangle$. In general, cdf-matching performed best in terms of most goodness-of-fit measures (RMSE-based and $R$ ), however at the expense of at least 2 more parameters in the transformation operator compared to the other statistical correction methods in the time domain. A transformation in the frequency domain through the low frequency resolution, but accurate and general, transfer function obtained by Welch's method yielded good results, but only rarely outperformed all other methods.

Because SCAN data are widely used in the United States, Table 2 is included to illustrate the expressions for upscaling the point data from the Powder Mill SCAN site (just outside field $D$ of the $O P E^{3}$ site) to the $\mathrm{OPE}^{3}$ average soil moisture by the statistical corrections methods $(M 1, M 3, M 4)$ in the time domain.

\section{Conclusions}

The objective of this study was to estimate the $\mathrm{OPE}^{3}$ field average soil moisture by using point observations, while attempting to limit the observational representativeness error due to the typical scale mismatch between point observations and modeling units (model initialization, calibration and validation) or remote sensing fields of view (calibration and validation of remote sensing data). Therefore, two approaches were considered: (i) identify locations which provide representative observations for the whole field, and (ii) identify relationships to upscale point data to render them representative for the spatial mean soil moisture. If a representative site is found, it might be useful to scale up the point data to get a further improved estimate of the pixel or field average soil moisture.

Representative locations at different soil layers were determined through calculation of the mean relative differences between point measurements and spatially averaged soil moisture over the entire $\mathrm{OPE}^{3}$ site. It was possible to indicate point locations which measure spatially representative soil moisture values at a single depth only, but it was impossible to find a probe which recorded measurements

Table 2 Conversion factors to scale up point measurements from the SCAN site $\left(u_{j, i}\right.$, with $j$ referring to the SCAN location) to $\mathrm{OPE}^{3}$ field averaged soil moisture $\left(\hat{y}_{i}\right)$ at different depths

\begin{tabular}{|c|c|c|c|c|c|c|c|}
\hline \multirow[t]{2}{*}{ Method } & \multirow{2}{*}{$\begin{array}{l}\text { Absolute difference } \\
\hat{y}_{i}=u_{j, i}-\left\langle d_{j}\right\rangle \\
\left\langle d_{j}\right\rangle\end{array}$} & \multicolumn{2}{|c|}{$\begin{array}{l}\text { Linear relationship } \\
\hat{y}_{i}=a+b u_{j, i}\end{array}$} & \multicolumn{4}{|c|}{$\begin{array}{l}\text { Cdf-matching } \\
\hat{y}_{i}=u_{j, i}+\left(p_{0}+p_{1} u_{j, i}+p_{2} u_{j, i}^{2}+p_{3} u_{j, i}^{3}\right)\end{array}$} \\
\hline & & $a$ & $b$ & $p_{0}$ & $p_{1}$ & $p_{2}$ & $p_{3}$ \\
\hline $10 \mathrm{~cm}$ & -11.83 & 14.66 & 0.75 & 10.88 & 0.74 & -0.067 & 0.0013 \\
\hline $20 \mathrm{~cm}$ & -15.14 & 17.52 & 0.75 & 6.32 & 2.94 & -0.273 & 0.0073 \\
\hline $50 \mathrm{~cm}$ & -18.04 & 23.02 & 0.47 & -8.14 & 8.52 & -0.843 & 0.02567 \\
\hline $100 \mathrm{~cm}$ & -3.14 & 10.30 & 0.57 & -42.43 & 3.68 & 0.050 & -0.00623 \\
\hline
\end{tabular}


that were representative of the spatial mean soil moisture at all soil layers. However, some probes could be identified with observations which were close to representing all layers well. De Lannoy et al. (2007) show a clear benefit of assimilating data from such probes for soil moisture initialization and parameter estimation for field averaged soil moisture simulation with a land surface model. Due to the complex geohydrology of the field, it was impossible to link the representativeness of individual probes to terrain features. This shows that the practical identification and use of representative sites would be difficult in areas where the variability in hydrological variables cannot be easily related to terrain characteristics.

Different statistically based methods were explored for the upscaling of point observations to spatial mean values, ranging from simple addition of a constant mean difference term, over a 2-parameter linear relationship to cdf-matching. The latter two methods yielded the best results. Expressions for these techniques are provided to upscale point data from the Powder Mill SCAN site to the OPE ${ }^{3}$ field. An upscaling in the frequency domain was effective, but other methods mostly performed better. Also, the spectrum-matching method should not be used for deeper soil layers, where only limited cyclic (e.g., diurnal) variation is found. Use of an autoregressive moving average filter for upscaling was most computationally expensive to identify and to apply.

Because it is very difficult to properly select representative locations within a satellite footprint or a modeled grid cell, the transformation or upscaling of point data is probably the only viable method to achieve data with limited representativeness error. When a point measurement is taken to represent a spatial mean value, then the accuracy of this point measurement will decrease and some systematic observational error may be introduced for its use at the coarser scale. Bias in the observations due to a scale mismatch can mostly be suppressed, i.e., the precision of the measurements can be largely improved, if the transformation is properly chosen. However, the accuracy of the upscaled observational information will be less than that of the point measurement. These issues of precision (bias) and accuracy (uncertainty) are important when data and model simulations are combined through an optimal weighting based on their respective degree of uncertainty in a data assimilation scheme.

\section{Acknowledgements}

The authors thank the Beltsville Agricultural Research Center (BARC) - Agricultural Research Service (ARS) of the USDA for collecting and providing the dataset. The Hydrological Sciences Branch (HSB) of NASA/GSFC is thanked for hosting the first author during part of the research. We also thank Michaël J. M. M. Steenbergen from Delft University of Technology, Faculty of Civil Engineering and Geosciences, for his support in the frequency domain analysis. The research is supported by a Ph.D.-fund of the Bijzonder Onderzoeksfonds (BOF) of the Ghent University. The anonymous reviewers are thanked for their constructive comments.

\section{References}

Atlas, D., Rosenfeld, D., Wolff, D.B., 1990. Climatologically tuned reflectivity-rainrate relations and links to area-time integrals. Journal of Applied Meteorology 29, 1120-1135.

Barling, R.D., Moore, I.D., Grayson, R.B., 1994. A quasi-dynamic wetness index for characterizing the spatial distribution of zones of surface saturation and soil water content. Water Resources Research 30 (4), 1029-1044.

Bindlish, R., Barros, A.P., 2002. Sub-pixel variability of remotely sensed soil moisture: An inter-comparison study of SAR and ESTAR. IEEE Transactions on Geoscience and Remote Sensing 40, 326-337.

Charpentier, M.A., Groffman, P.M., 1992. Soil moisture variability within remote sensing pixels. Journal of Geophysical Research 97, 18987-18995.

Chen, Y., 2006. Letter to the editor on Rank Stability or Temporal Stability. Soil Science Society of America Journal 70 (1), 306.

Cosh, M.H., Jackson, T.J., Bindlish, R., Prueger, J.H., 2004. Watershed scale temporal and spatial stability of soil moisture and its role in validating satellite estimates. Remote Sensing of the Environment 92, 427-435.

Crow, W.T., Ryu, D., Famiglietti, J.S., 2005. Upscaling of field-scale soil moisture measurements using distributed land surface modeling. Advances in Water Resources 28, 1-14.

De Lannoy, G.J.M., Verhoest, N.E.C., Houser, P.R., Gish, T., Van Meirvenne, M., 2006. Spatial and temporal characteristics of soil moisture in an intensively monitored agricultural field $\left(\mathrm{OPE}^{3}\right)$. Journal of Hydrology 331 (3-4), 719-730.

De Lannoy, G.J.M., Pauwels, V.R.N., Houser, P.R., Gish, T., Verhoest, N.E.C., 2007. Representativeness of point soil moisture observations, upscaling and assimilation. In: Quantification and Prediction of Predictive Uncertainty for Sustainable Water Resources Management(Proceedings of Symposium HS2004 at IUGG2007), vol. 313. IAHS Publ., Perugia, Italy, pp. 249-257.

Drusch, M., Wood, E.F., Gao, H., 2005. Observation operators for the direct assimilation of TRMM microwave imager retrieved soil moisture. Journal of Geophysical Research 32, L15403.1L15403.4.

Famiglietti, J.S., Devereaux, J.A., Laymon, C.A., Tsegaye, T., Houser, P.R., Jackson, T.J., Graham, S.T., Rodell, M., van Oevelen, P.J., 1999. Ground-based investigation of soil moisture variability within remote sensing footprints during the Southern Great Plains 1997 (sgp97) hydrology experiment. Water Resources Research 35 (6), 1839-1851.

Gish, T.J., Dulaney, W.P., Kung, K.-J.S., Daughtry, C., Doolittle, J.A., Miller, P.T., 2002. Evaluating use of ground-penetrating radar for identifying subsurface flow pathways. Soil Science Society of America Journal 66 (5), 1620-1629.

Gómez-Plaza, A., Alvarez-Rogel, J., Castillo, V.M., 2000. Spatial patterns and temporal stability of soil moisture across a range of scales in a semi-arid environment. Hydrological Processes 14, 1261-1277.

Grayson, R.B., Western, A.W., 1998. Towards areal estimation of soil water content from point measurements: Time and space stability of mean response. Journal of Hydrology 207 (1-2), 68-82.

Jacobs, J.M., Mohanty, B.P., Hsu, E.-C., Miller, D., 2004. SMEX02: Field scale variability, time stability and similarity of soil moisture. Remote Sensing of Environment 92, 436-446.

Martínez-Fernández, J., Ceballos, A., 2003. Temporal stability of soil moisture in a large-field experiment in Spain. Soil Science Society of America Journal 67, 1647-1656.

Martínez-Fernández, J., Ceballos, A., 2005. Mean soil moisture estimation using temporal stability analysis. Journal of Hydrology $312(1-4), 28-38$.

Mohanty, B.P., Skaggs, T.H., 2001. Spatio-temporal evolution and time-stable characteristics of soil moisture within remote sensing footprints with varying soil, slope and vegetation. Advances in Water Resources 24, 1051-1067. 
Oppenheim, A.V., Schafer, R., 1975. Digital Signal Processing. Prentice-Hall, Englewood Cliffs, NJ.

Pachepsky, Y.A., Guber, A.K., Jacques, D., 2005. Temporal persistence in vertical distributions of soil moisture contents. Soil Science Society of America Journal 69 (2), 347-352.

Reichle, R.H., Koster, R., 2004. Bias reduction in short records of satellite soil moisture. Geophysical Research Letters 31, L19501.1-L19501.4.

Vachaud, G., De Silans Passerat, A., Balabanis, P., Vauclin, M., 1985. Temporal stability of spatially measured soil water probability density function. Journal of the Soil Science Society of America 49, 822-827.

Van Pelt, R.S., Wierenga, P.J., 2001. Temporal stability of spatially measured soil matrix potential probability density function. Soil Science Society of America Journal 65, 668677.

Welch, P.D., 1967. The use of fast Fourier transform for the estimation of power spectra: A method based on time averaging over short modified periodograms. IEEE Transactions on Audio and Electroacoustics AU-15, 70-73. 